INPLASY

PROTOCOL

To cite: Xue et al. Clinical efficacy and safety of moxibustion in the treatment of chronic fatigue syndrome: a systematic review and metaanalysis. Inplasy protocol

202140031. doi:

10.37766/inplasy2021.4.0031

Received: 06 April 2021

Published: 06 April 2021

Corresponding author:

Kaiyang Xue

2317649912@qq.com

Author Affiliation:

Evidence-based Medicine

Research Center, Guizhou

University of Traditional

Chinese Medicine

Support: No: 3040-040200104.

Review Stage at time of this submission: The review has not yet started.

Conflicts of interest:

None declared.

\section{Clinical efficacy and safety of moxibustion in the treatment of chronic fatigue syndrome: a systematic review and meta- analysis}

Xue, K1; Wang, Y2; Wang, X3; Chen, P4; Xiao, C5; Fu, J6; Cui, J7.

Review question / Objective: Is moxibustion effective and safe in the treatment of chronic fatigue syndrome?

Condition being studied: Chronic Fatigue syndrome, CFS. Information sources: We will systematically search PubMed, EMBASE, Cochrane library, Sinomed, the CNKI (Chinese National Knowledge Infrastructure), VIP (Chinese Scientifific Journals Database), Wanfang Database, with ClinicalTrials.gov and Chinese Clinical Trial Registry.

INPLASY registration number: This protocol was registered with the International Platform of Registered Systematic Review and Meta-Analysis Protocols (INPLASY) on 06 April 2021 and was last updated on 06 April 2021 (registration number INPLASY202140031).

\section{INTRODUCTION}

Review question / Objective: Is moxibustion effective and safe in the treatment of chronic fatigue syndrome?

Rationale: Chronic fatigue syndrome (chronic Fatigue syndrome, CFS) is a worldwide disease of unknown cause, which is characterized by long-term extreme fatigue for more than 6 months, accompanied by inattention, memory loss, sleep disorders and many other nonspecific symptoms.Chronic fatigue significantly reduces the quality of life of patients and even may induce dysfunction, which has become one of the most severe public health problems in the world.Modern 
medicine mainly supports the treatment of CFS such as anti-anxiety, immune regulation, and nutritional supplementation, but the effectiveness is still controversial.Studies have shown that moxibustion can effectively improve fatigue symptoms and improve CFS-related sleep quality, pain and paresthesia, gastrointestinal dysfunction and other symptoms.Therefore, the purpose of this study is to explore the efficacy and safety of moxibustion in the treatment of CFS by pooling the current randomized controlled trials, in order to provide high-quality clinical evidence.

Condition being studied: Chronic Fatigue syndrome, CFS.

\section{METHODS}

Search strategy: We will systematically search 7 major databases (PubMed, EMBASE, Cochrane library, CNKI, VIP, SinoMed and Wanfang Database) and collect the RCTs of moxibustion treatment of CFS. We will use a combination of medical subject words and free words to search for terms such as "Chronic Fatigue syndrome", " chronic Fatigue ", "and Moxibustion".For including more studies, we will also search the two trial registration platforms ClinicalTrials.gov and Chinese Clinic Trials.gov, as well as references from related reviews.

Participant or population: Patients with CFS, without restrictions on gender, age, course of disease, severity and source of cases.

Intervention: Moxibustion that ignites moxa and produces moxa smoke and temperature is the intervention measure concerned in this study. We do not restrict the production methods of moxa,nor the types of moxibustion (direct moxibustion, indirect moxibustion), but electronic moxibustion will be excluded. The experimental group will be included in the use of moxibustion alone or in combination with other basic treatments VS same basic treatment CFS, basic treatment including acupuncture, Chinese herbal medicine, medicine, medicine, placebo-treated like the RCTS.

Comparator: The comparator should be the same basic treatment as the intervention group.

Study designs to be included: Randomized controlled trials.

Eligibility criteria: We will include patients according to the criteria of the US CDC definition (1988 or 1994 ) for CFS.

Information sources: We will systematically search PubMed, EMBASE, Cochrane library, Sinomed, the CNKI (Chinese National Knowledge Infrastructure), VIP (Chinese Scientifific Journals Database ), Wanfang Database, with ClinicalTrials.gov and Chinese Clinical Trial Registry.

Main outcome(s): The main outcome is Fatigue Assessment Instrument ( FAI ).

Additional outcome(s): Fatigue Scale -14 (the FS-14), Fatigue Severity Scale (the FSS), Pittsburgh Sleep Quality Index (PSQI), natural killer (NK) Cells, interleukin 2 (IL-2), T lymphocyte subsets (CD4+, CD8 +), recovery rate, total effective rate, cure rate and adverse reactions.

Data management: 1) Publishing features: title, first author, establishments, journals, and publication date; 2) patients and treatment: sex, age, source of the patients, sample size, with or without blinding, CFS Moxibustion site, moxibustion strength, duration of treatment, adverse reactions, adverse events, follow-up time, loss rate and reasons; 3) Outcome data: baseline and follow-up measurement of each index.

Quality assessment / Risk of bias analysis: Two reviewers will independently and repeatedly assess the risk of bias in the included studies and cross-check the results. The disagreement will be determined by a third reviewer. The risk of bias will be assessed using the risk assessment tool for randomized controlled trial recommended in the Cochrane handbook 5.1.0. It includes 7 items: 1) 
random list generation method; 2) allocation concealment; 3) blinding of patients and clinicians; 4) blinding of outcome evaluator; 5) data completeness; 6) selective reporting; 7) other bias sources. Each item will be rated as low, high, or uncertain risk of bias.

Strategy of data synthesis: 1) Meta-analysis for continuous outcomes: weighted mean difference or standardized mean difference and $95 \%$ confidence intervals (Cls) will be used as the effect measures and the inverse variance method will be used to combine the data; 2) Meta-analysis for dichotomous outcomes: relative risks and $95 \% \mathrm{Cls}$ will use as the effect measures and the data will be combined using the Mantel-Haenszel method. Cochran's $Q$ test and 12 statistics will be used to evaluate the heterogeneity among studies in the metaanalysis.

Subgroup analysis: Based on the experience of moxibustion therapy in clinical application, when there is obvious heterogeneity in the analysis results, we will analyze the source of the heterogeneity from the following subgroups: 1) Subgroup analysis by moxibustion type: The randomized controlled trial of indirect moxibustion was compared with that of direct moxibustion. It is expected that the former has better curative effect. 2) Subgroup analysis by treatment course: We will compare randomized controlled trials with a course of treatment $>2$ weeks and $\leq$ 2 weeks. The former is expected to have a better effect.

Sensitivity analysis: We will conduct the following two sets of sensitivity analyses: 1) excluding studies with high risk of bias; 2) using a fixed-effects model to collect the data from the meta- analysis.

Language: No restrictions.

Country(ies) involved: China.

Keywords: moxibustion, chronic fatigue syndrome, protocol, systematic review, meta-analysis.
Dissemination plans: We aim to publish the results of this systematic review in a peerreviewed journal.

Contributions of each author:

Author 1 - Kaiyang Xue - Methodology; Writing: original draft.

Email: 2317649912@qq.com

Author 2 - Yanping Wang - Methodology; Writing: original draft.

Author 3 - Xianzhu Wang - Investigation.

Author 4 - Pingnan Chen - Investigation.

Author 5 - Caihong Xiao - Investigation.

Author 6 - Jing Fu - Conceptualization; Writing: original draft; Writing: review \& editing.

Author 7 - Jin Cui - Funding acquisition; Writing: review \& editing. 